\title{
Short term effects of restricted food availability and peripheral leptin injections in redheaded bunting, Emberiza bruniceps
}

\author{
Neelu Jain Gupta* \\ Department of Zoology, Chaudhary Charan Singh University, Meerut- 250004 (Uttar Pradesh), \\ India \\ Samya Das \\ Department of Zoology, Chaudhary Charan Singh University, Meerut- 250004 (Uttar Pradesh), \\ India \\ Anit Kumar \\ Department of Zoology, Chaudhary Charan Singh University, Meerut- 250004 (Uttar Pradesh), \\ India \\ Sanjay Kumar Bhardwaj \\ Department of Zoology, Chaudhary Charan Singh University, Meerut- 250004 (Uttar Pradesh), \\ India \\ Nidhi Yadav \\ Department of Zoology, Chaudhary Charan Singh University, Meerut- 250004 (Uttar Pradesh), \\ India \\ ${ }^{*}$ Correspondence author. Email: drneelujgupta@hotmail.com
}

\section{How to Cite}

Gupta, N. J. et al. (2021). Short term effects of restricted food availability and peripheral leptin injections in redheaded bunting, Emberiza bruniceps. Journal of Applied and Natural Science, 13(4), 1430 - 1436. https://doi.org/10.31018/jans.v13i4.3139

\begin{abstract}
Migratory birds need continued food supply and efficient metabolic machinery to meet high energy demands of the magnanimous feat of flight. Two questions are important i.e. as to 1) how a bird adapts to a temporary food constrain on a daily basis, and 2) how peripheral leptin, an anorectic hormone, impacted feeding and migratory behaviour in buntings? The aim of this study was to induce a non-photoperiodic tweak in the physiology of redheaded buntings through exogenous leptin administration and study its effect on their food intake and migratory behaviour. Groups of male redheaded buntings, Emberiza bruniceps $(n=17)$ were transferred from short (8L: 16D) to long (16L: 8D) days and presented with food only either for first (morning food presence, MFP) or second (evening food presence, EFP) half of the $16 \mathrm{~h}$ lighted phase, while control group received food ad libitum. Total daily food intake (FI) did not differ significantly between the MFP, EFP and controls, but hourly FI in MFP and EFP indicated increased activity differences based on time of food availability and bird's tendency to cache food/ recompense for food scarcity during migration. In another experiment, a chemical tweak in bird's FI was induced by peripheral administration of leptin, to add to current understanding of transition in buntings' metabolic efficiency during high energy demanding migratory journey. Exogenous leptin appeared to safeguard cadaveric effect of exogenous injection in migrating buntings through promoting blood cholesterol and reduced liver fibrosis. Food restriction in the morning was better responded by buntings than that in evening. Therefore, migratory buntings exhibited diurnal variation in response to food scarcity.
\end{abstract}

Keywords: Activity, Food, Leptin, Migration, Restriction

\section{INTRODUCTION}

Increasing day length and higher food consumption are two major determiners that trigger migration in night migratory songbirds (Jain and Kumar, 1995; McWilliams et al., 2004). Food consumption is directly affected by food availability in nature (Klaassen, 1996). Natural daylength changes are predictable, but food availability may get constrained in nature at any point in time, thus adversely affecting migration (Ferretti et al., 2019). It was hypothesized that buntings have the ability to sense the food constraints and conscientiously increase hourly consumption of food to makeover for the ensuing scarcity of food. In other words, hourly allocation of food consumption in the form of daily food eating patterns (Morton, 1967) is as important as total daily food intake (FI). It is suggested that differential food consumption at different times of day might be 
proportionately related to differential food availability besides the migratory state of the birds. Food-searching behaviour is aggravated by food restriction, which is observed as hyperactivity in caged birds (Lynn et al., 2003; Krause et al., 2017). It is important to understand the daily profile of $\mathrm{FI}$ of migratory birds during migration and how they tend to recompense food scarcity (or limited food availability) at different times of the day. In addition to food availability in nature, birds' ability to cache food (store food in the crop for longer duration) also affects their daily pattern of food consumption (Prabhat et al., 2020).

Birds' satiety is also important in deciding the metabolic consequence of food availability. Leptin is an adipose tissue secreted protein satiety hormone in mammals. It is not very well reported in birds, although the presence of its receptors in migratory passerines is reported (Kochan et al., 2006), which is supported by investigations involving exogenous injections and its anorectic impact in migratory birds during non-migratory lifehistory stage (Cerasale et al., 2011; Hen et al., 2008). Higher leptin level in mammals inhibits de novo fatty acid synthesis and increase the release and utilization of free fatty acids from adipose tissue (William et al., 2002). The injected leptin would be interpreted as a mammalian analogue, different from avian leptin in buntings, yet it is implicated in birds physiology, including growth. The high energy demanding events, such as migration, need prior fat accumulation to fuel migratory flight; these excess fat stores lead to obesity and might reduce leptin sensitivity similar to that in mammals (Myers et al., 2010) or differently depending on the result birds metabolic phenotype. For example, studies on white-throated sparrows showed that exogenous leptin facilitates fat deposition and maintains high $\mathrm{FI}$ rate in migrating sparrow but not in wintering sparrow (Cerasale et al., 2011).

In mammals, leptin is secreted from adipocytes, affects feeding, and acts as a signal between peripheral energy depots and the central nervous system (Lõhmus and Björklund, 2009). In mammals, leptin complements longterm regulation of appetite through its adipocytic secretion, but in birds, leptin operates in the short term regulation of appetite based on the digestive activity (Seroussi et al., 2019). Further to this, maintenance of energy balance in migratory birds is most intriguing because these undergo a fat accumulation-depletion cycle to meet the high energy demands of migration (Goymann et al., 2017). Exogenous leptin administration affects migrating birds, but these effects vary among different birds. Peripherally injected leptin reduces mass gain in the migrating coal tits (Henderson et al., 2018), while in Adelie penguin and bar-tailed godwits, there is lack of leptin activity in blood (Yosefi et al., 2010).

Environmental non-photoperiodic factors like temperature, humidity, wind direction (Rees, 1982) have been studied in bird migration, besides physiological factors such as involvement of reproductive organs (Gupta and Kumar, 2013) and exogenous drug administration (Fusani et al., 2013). Taken together, investigations involving food restriction and peripheral leptin injections are intended to further enrich our current understanding of hormonal axis regulating appetitive and consummatory behaviour of migrating redheaded bunting in two ways. Firstly, stimulating peptide hormone ghrelin release from proventriculus, through restricted feeding (Kaiya et al., 2009) and secondly, test bird's response to peripheral leptin administration in migratory bunting (Boswell and Dunn, 2015). Our purpose of exogenous leptin administration in buntings was to induce a nonphotoperiodic tweak in their $\mathrm{FI}$, to test its effect on migratory behavior.

\section{MATERIALS AND METHODS}

This study was conducted on the migratory adult male red-headed buntings (Emberiza bruniceps), family Emberizidae, in accordance with Institutional Animal Ethics Committee (IAEC) guidelines of Chaudhary Charan Singh University, Meerut, India. Buntings were brought and maintained in laboratory conditions, as reported earlier (Das and Gupta, 2016). Two experiments were performed. In experiment one, involving food restriction, buntings were individually held under short days $(n=17,8 L: 16 D$; $L=300$ lux, $D=0.3$ lux; where $n$ sample size, L-light, D- dark), and transferred to long days; $16 \mathrm{~L}: 8 \mathrm{D}$; $\mathrm{L}=300$ lux, $\mathrm{D}=0.3$ lux) to photo-induce migratory restlessness. For the first three long days, food was provided ad libitum. Then, continuing all birds under long days, food availability timings were restricted to 8 of the 16 lighted hours. Food was available in these two food restriction test groups, either $8 \mathrm{~h}$ from lights on (MFP- Morning Food Presence) or $8 \mathrm{~h}$ up to lights off (EFP- Evening Food Presence). Besides EFP and MFP groups, the third control group received unrestricted food supply. This food administration routine continued for five LD cycles, during which $\mathrm{Fl} /$ bird/day and locomotor activity was measured. In another experiment, night migratory restlessness was photo-induced in birds $(n=10)$ held under long days. Once in migratory state, buntings were intramuscularly injected either leptin (Recombinant murine leptin, Peprotech, USA, $n=5$ ) or vehicle (Phosphate Buffer Saline PBS, $n=5$ ) in the pectoralis major twice daily for five days at a dose of 20 $\mu \mathrm{g}$ per bird dissolved in $100 \mu \mathrm{l}$ PBS as prescribed by Cerasale et al. (2011). Blood glucose, uric acid and cholesterol were measured four-hourly using EasyTouch GCU (Glucose Cholesterol Uric acid) MultiFunction Monitoring System. Body vitals of all birds, i.e. weight, fat score, behavioural observations of two hourly/daily FI, daily activity rest, and histological observations of liver tissue, were made. 
The activity of individually caged birds was recorded using data-logging system- Chronobiology Kit (Stanford Software System, USA) system (Malik et al., 2004).

The data were represented as mean \pm SEM and analysed using " $t$ " test or One Way ANOVA as applicable. Significance was taken on $P<0.05$. Statistical analysis was carried out using GraphPad Prism software (version 3.0 La Jolla, CA, USA)

\section{RESULTS}

Migratory buntings recompense time restrictions in food availability

Fig.1 (a-c) depicts double plotted actogram of representative birds of MFP, EFP and control groups respectively. The control group (16L: 8D; food ad libitum) bunting exhibited significantly higher $\left(t_{6}=14.81 ; P<\right.$ 0.05 ) activity, Fig.1 (f) throughout the light phase, whereas birds were apparently but not significantly $\left(t_{3}=1.976\right.$; $\left.P>0.05\right)$ Fig.1 (d), more active during food absence in MFP but significantly $\left(t_{3}=9.307 ; P<0.05\right)$ Fig.1 (e) more active during food absence in EFP groups. Overall, daily $\mathrm{FI}$ did not vary significantly among MFP, EFP and control groups $\left(F_{2,21}=1.387\right.$; $P>$ 0.05 ); but daily $\mathrm{FI}$ per hour (total $\mathrm{Fl} /$ number of hours of food availability), $\left(F_{2,21}=4.89 ; \mathrm{P}<0.05\right)$. Fig.1 (g, h) shows daily and hourly $\mathrm{FI}$ of each of the groups, respectively. EFP birds exhibited higher hourly food consumption $\left(t_{4}=2.510 ; P<0.05\right)$ than MFP. As shown by the bar size of the testicular volume in representatives of MFP and EFP groups in Fig.1 (i), birds compromised gonadal growth.

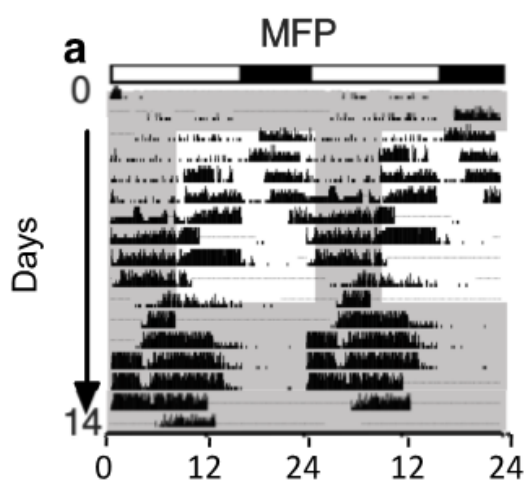

b
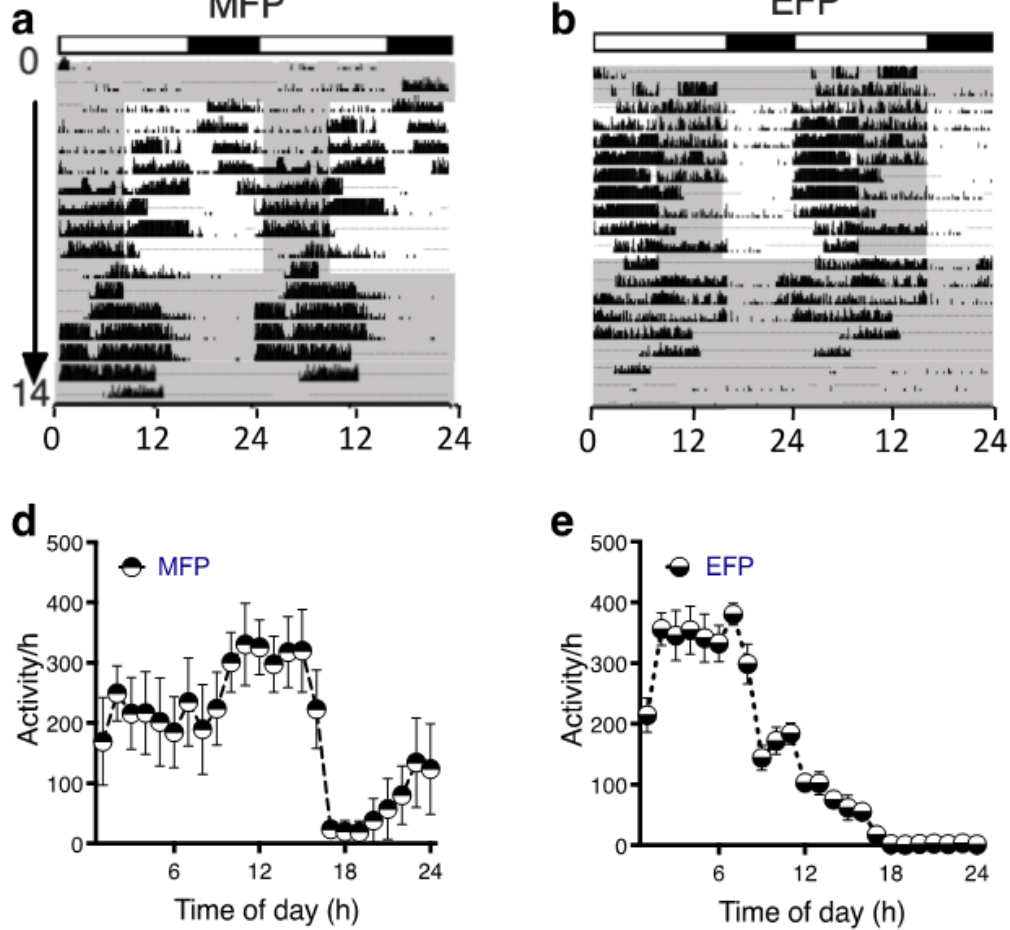

g

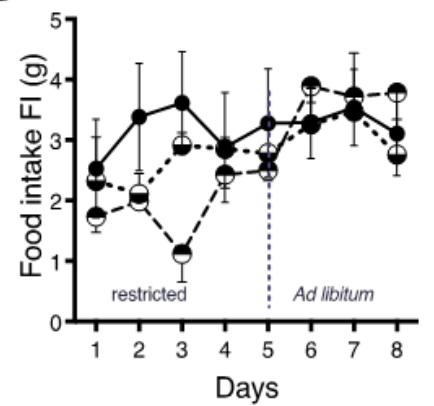

$\mathbf{e}$

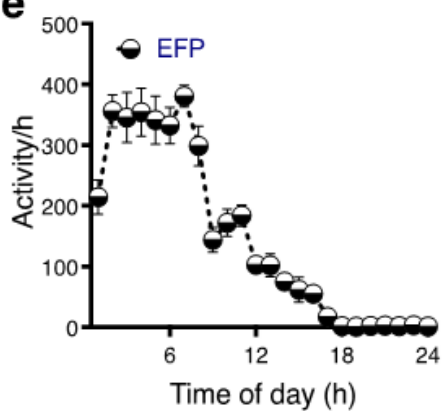

h

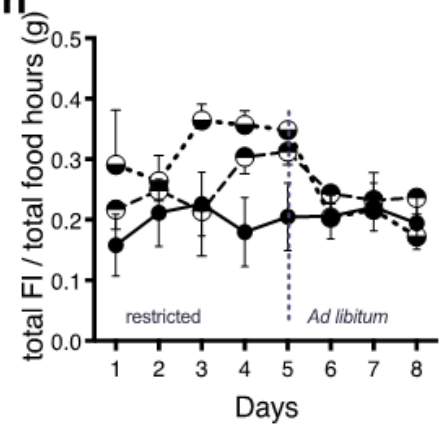

c
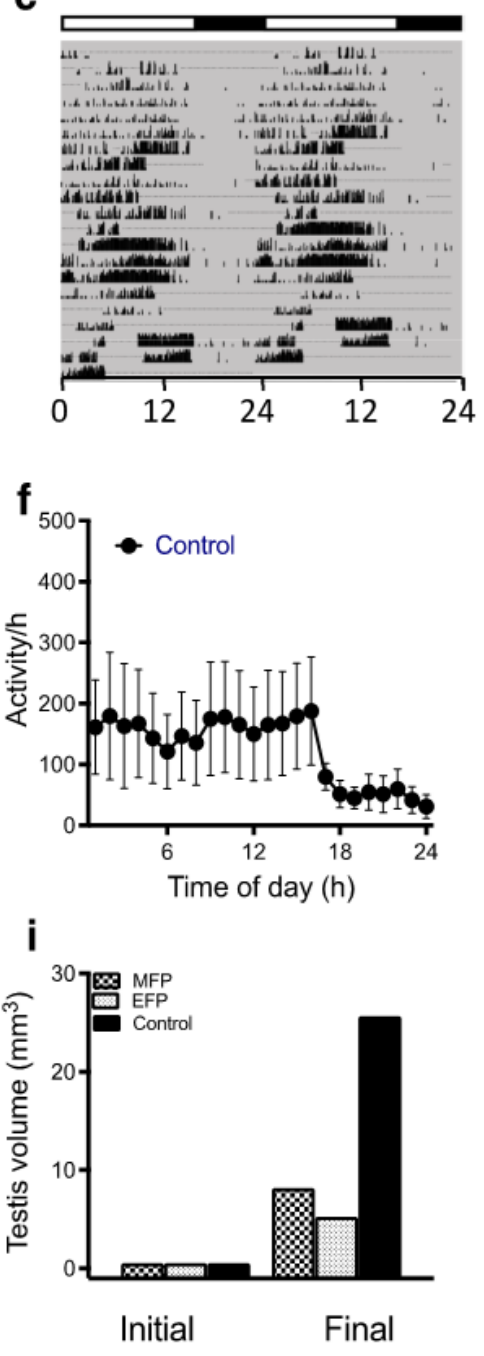

Fig. 1 a-i. Double plotted actogram for representative male redheaded bunting transfer to long days (16L: 8D 300 Lux, 0.3 Lux). Grey shaded area indicates food availability, where light and dark phases are shown by LD bar. (a): MFP (Morning Food Presence, ZTO- ZT8 where ZTO- lights on); (b): EFP (Evening Food Presence, ZT8- ZT16 where ZTOlights on); (c): Food provided ad libitum (Control). (d, e, f): average daily profile of activity of groups MFP, EFP and control respectively (mean \pm SEM). $(g, h)$ : daily and hourly food intake; for MFP (upper closed circles), EFP (lower closed circles), and controls (closed circles), and (i): testis volume of representative bird of MFP, EFP and control group 


\section{Leptin injection safeguards cadaveric effects in migrating buntings}

Double plotted actograms of representative migrating buntings injected with leptin or vehicle, are shown in Fig. $2(a, b)$. All birds were night active before injection Fig. 2 (c), which was discontinued during Fig. 2 (d), and after Fig. 2 (e) leptin/vehicle treatment. No significant variation in activity was observed in birds subjected to exogenous leptin/vehicle administration. Although, in both groups, there was an apparent decline in body mass Fig. 2 (f), fat score Fig. 2 (g) and utilization efficiency Fig. 2(h); leptin treated buntings maintained better relative mass (than vehicle treated birds). FI declined in all injected birds and did not differ among leptin or vehicle groups Fig. 2 (I). Albeit leptin treated birds exhibited comparatively lesser decline in body mass (leptin: $t_{4}=8.107, p=0.013$; vehicle: $t_{4}=9.844, P<0.001$ ). Serum glucose levels (ranging between $400-480 \mathrm{mg} / \mathrm{dl}$ ) of leptin treated birds were lower than vehicle treated birds Fig. 2 (i). Exogenous leptin administration had no effect on blood uric acid Fig.2 (j). Blood cholesterol (raging between 150-300 mg/dl) level of leptin-treated but not vehicle-treated birds, dropped within an hour of injection Fig. 2 (k). There was time of day-difference in serum glucose and cholesterol levels. Liver fibrosis was reduced in leptin treated birds as indicated by significantly lesser $\left(t_{8}=3.965, P<0.01,2 M\right)$ cell density. Student's paired t-test revealed a significant decline in fat score of vehicle treated Fig.1 (g) birds, but not in leptin treated birds; leading to better activity-fat score correlation $(r=0.93 ; \quad P<0.02$, Spearman correlation, Fig. $2(g, n)$ in leptin than vehicle injected birds.

\section{DISCUSSION}

The daily $\mathrm{FI}$ adjustments are associated with energy requirements in migratory birds (Bairlein, 1990). Food availability in nature is a potential cue as compared to

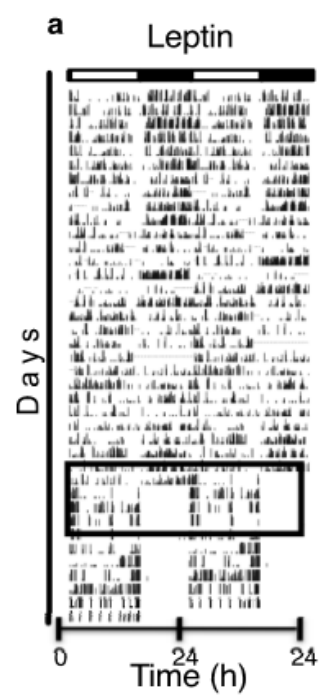

I

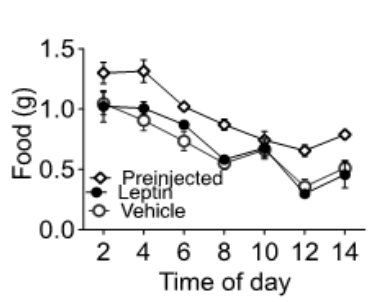

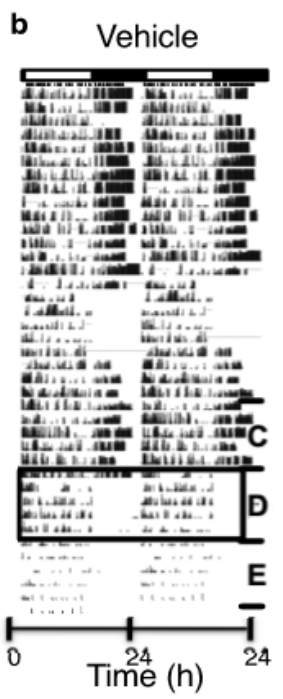

m

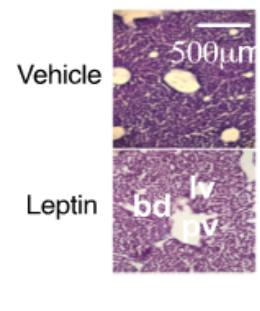

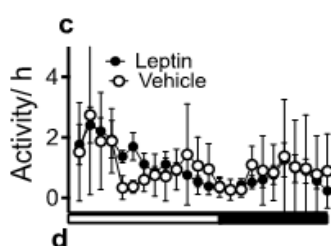
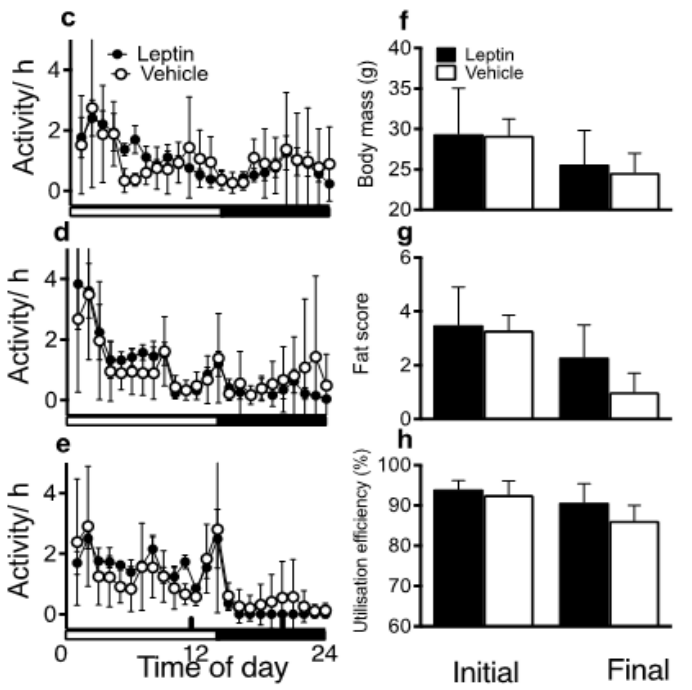

n

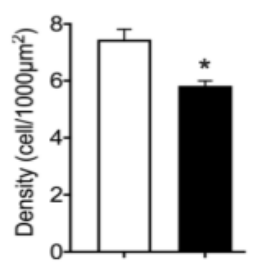

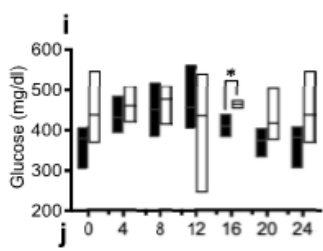
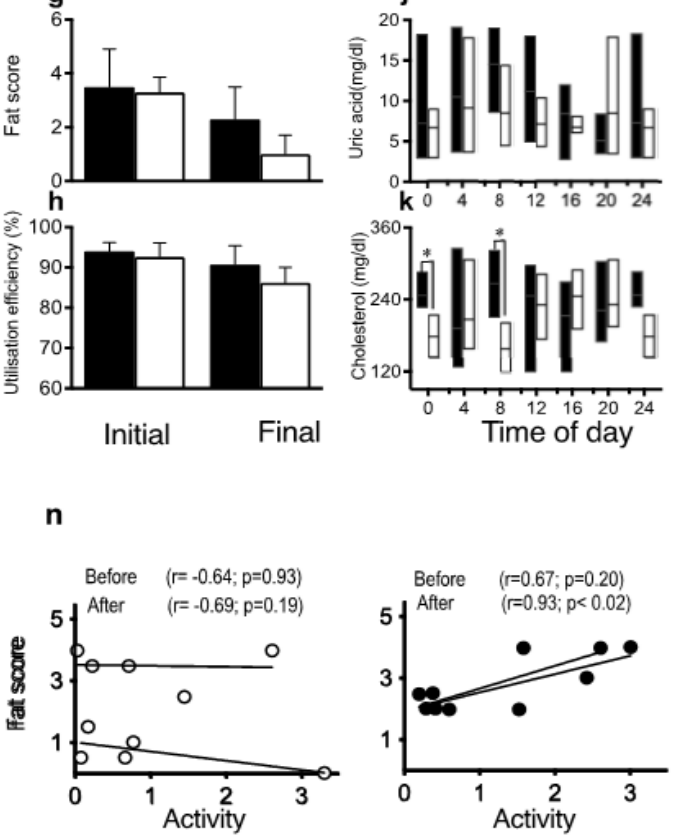

Fig. 2 a-n. Double plotted actogram for representative male redheaded bunting injected with leptin and vehicle (PBS) respectively. a, b): Bar above the actogram represents light: dark (14L: 10D 300:0.3 Lux), (c, d, e): show average daily profile of activity of leptin (closed circle) and vehicle (open circle) treated buntings, before, during and after injection, respectively (mean \pm SEM). ( $f, g, h)$ : body mass fat score and utilization efficiency of leptin (closed bars) or vehicle (open bars) treated buntings before and after injection respectively. (i, $j, k)$ : scatter plot showing glucose, uric acid and cholesterol levels in blood of leptin (closed bars) or vehicle (open bars) treated buntings after injection. (l): Daily food eating pattern of pre-injected (small open circle), leptin (closed circle) and vehicle (open circle) injected groups. (m): representative micrographs of hematoxylin-eosin H\&E stained liver sections showing cell density (cells/1000 $\mu m^{2}$ ) in vehicle (upper) or leptin (lower) treated birds. Abbreviations- bd: bile duct, Iv: lymph vein, pv: portal vein. (n): correlation between fat score- activity of vehicle and leptin birds 
photoperiod in non-migratory birds, such as zebra finch (Perfito et al., 2008; Misra, 2016). Timing and duration of food availability affects photoperiodic induction of gonadal recrudescence, is suggested in the blackheaded bunting (Kumar et al., 2001), house sparrow (Bhardwaj and Anushi, 2004) and redheaded bunting (Budki et al., 2009). Although food deprivation in the laboratory may not equate to restricted availability or food scarcity in nature, birds tend to recompense shortage of food availability. Our study corroborates with this hypothesis and suggests that buntings might achieve success in compensating for food scarcity during some hours of day, by consuming more food, when available (Fig. 1a, b, d and e). Interestingly, total daily FI was not significantly $\left(F_{(2,21)}=1.38, P=0.27\right)$ different between the MFP, EFP and controls, but when analyzed in terms of food consumed per hour of food availability, there were differences in hourly $\mathrm{FI}$ among MFP and EFP which indicated differential caching of food and might explain altered activity pattern. Whereas this might appear as plasticity in their daily food eating pattern (Das and Gupta, 2016), activity behaviour might be invigorated by food absence immediately after lights 'on' in EFP group, which would be masked in MFP birds because they, (1) ate after night fasting, and (2) possibly cached food, to recompense evening shortage, thus exhibiting less activity difference. The latter, observed in finches (Prabhat et al., 2020), needs to be assessed for buntings. Food restriction was tested only for a very short span, i.e., five days, to enable birds to regain health; the data does not suggest long term effects such as those on energy states, testes size and body weight. One practical reason for this ad libitum food resumption was due to suspected mortality of small-sized birds, as 2 birds died in MFP and EFP groups. Despite limitations, our study is consistent with the evidence from food-time restriction studies in other animals and birds and insects, such as Drosophila (Gill and Panda, 2011) and songbirds (Kumar et al., 2001). While the study on time of food availability adds to our current understanding of birds' behavioural ability to make an adjustment to support extensive energy budget requisite during migration, it's metabolic adjustment could also be understood through a chemical tweak in its satiety, and thus its energy budgeting.

How leptin might be implicated in the regulation of energy balance in birds during migration is less explained. Long-distance migrating birds, increase the availability of energy stores, particularly fatty acids (stored in adipocyte), that are important for the preparation for imminent prolonged flight (Landys et al., 2005). Lõhmus and colleagues in 2003 suggested that exogenous leptin depressed $\mathrm{FI}$ in migratory great tits (Lõhmus et al., 2003). However, the ability of the adipocytes to endogenously support migration then became an aspect of the controversy surrounding avian leptin. Notably, extreme GC content in genes encoding avian adipokines recently suggested the implication of leptin in energy-balance control evolved differently in birds to suit their aerial lifestyles (Friedman-Einat and Seroussi, 2019). Liver fibrosis was reduced in leptin treated birds, suggesting decreased collagen/ inactivate hepatic stellate cells (Kurokawa and Ohkohchi, 2017).

Despite the similar decline in $\mathrm{FI}$ in treatment groups, upon injection, leptin treated birds recovered $\mathrm{FI}$ earlier than controls and exhibited a lesser decline in body weight within a few days after injection. Lower serum glucose levels and higher cholesterol correlate with high mass-specific metabolic rates and might indicate the downstream biochemical evidence to support that leptin is positively correlated with increased endurance in migratory birds. However, this needs to be proven with more robust biochemical assays involving high throughput proteomic/metabolomic analyses (Gupta et al., 2020).

\section{Conclusion}

The present study clearly showed that exogenous leptin safeguarded the cadaveric effect of 'injection-stress' in migrating buntings Emberiza bruniceps and lowered liver cell density, an indicative of decreased collagen/ inactivate hepatic stellate cells, thus promoting blood cholesterol and reduced liver fibrosis. The current study is important to highlight the adaptive nature of feeding physiology in migratory birds.

\section{ACKNOWLEDGEMENTS}

Authors acknowledge bird permission from Chief Wildlife Warden, Jaipur, Rajasthan. The financial assistance from the Science and Engineering Research Board (SERB), Department of Science and Technology, New Delhi [CRG/2019/002542 dated 27.02.2020] to Neelu Jain Gupta is gratefully acknowledged.

\section{Conflict of interest}

The authors declare that they have no conflict of interest.

\section{REFERENCES}

1. Bairlein, F. (1990). Nutrition and Food Selection in Migratory Birds. In: Gwinner E (Ed.) Bird Migration (pp 198-213). Berlin Heidelberg: Springer-Verlag

2. Bhardwaj, S. K. \& Anushi (2004). The effect of duration and time of food availability on the photoperiodic response in the male house sparrow, Passer domesticus. Reproduction, Nutrition, Development, 44(1), 29-35. doi.org/10.1051/rnd:2004014

3. Boswell, T. \& Dunn, I. C. (2015). Regulation of the avian central melanocortin system and the role of leptin. Gen- 
eral and Comparative Endocrinology, 221, 278-283. doi.org/10.1016/j.ygcen.2014.12.009

4. Budki, P., Rani, S. \& Kumar, V. (2009). Food deprivation during photosensitive and photorefractory life-history stages affects the reproductive cycle in the migratory Redheaded Bunting (Emberiza bruniceps). The Journal of Experimental Biology, 212(2), 225-230. doi.org/10.1242/ jeb.024190

5. Cerasale, D. J., Zajac, D. M. \& Guglielmo, C. G. (2011). Behavioral and physiological effects of photoperiodinduced migratory state and leptin on a migratory bird, Zonotrichia albicollis: I. Anorectic effects of leptin administration. General and Comparative Endocrinology, 174(3), 276-286. doi.org/10.1016/j.ygcen.2011.08.025

6. Das, S. \& Gupta, N. J. (2016). Seasonal modulation of diurnal food consumption in Indian songbirds. Biological Rhythm Research, 47(4), 621-629. doi.org/10.10 80/09291016.2016.1178415

7. Ferretti, A., Maggini, I., Lupi, S., Cardinale, M. \& Fusani, L. (2019). The amount of available food affects diurnal locomotor activity in migratory songbirds during stopover. Scientific Reports, 9(1), 19027. doi.org/10.1038/s41598019-55404-3

8. Friedman-Einat, M. \& Seroussi, E. (2019). Avian Leptin: Bird's-Eye View of the Evolution of Vertebrate EnergyBalance Control. Trends in Endocrinology and Metabolism: TEM, 30(11), 819-832. doi.org/10.1016/j.t em.2019.07.007

9. Fusani, L., Coccon, F., Rojas Mora, A. \& Goymann, W. (2013). Melatonin reduces migratory restlessness in Sylvia warblers during autumnal migration. Frontiers in Zoology, 10(1), 79. doi.org/10.1186/1742-9994-10-79

10. Gill, S. \& Panda, S. (2011). Feeding mistiming decreases reproductive fitness in flies. Cell Metabolism, 13(6), 613614. doi.org/10.1016/j.cmet.2011.05.003

11. Goymann, W., Lupi, S., Kaiya, H., Cardinale, M. \& Fusani, L. (2017). Ghrelin affects stopover decisions and food intake in a long-distance migrant. Proceedings of the $\mathrm{Na}$ tional Academy of Sciences of the United States of America, 114(8), 1946-1951. doi.org/10.1073/pnas.1619565114

12. Gupta, N. J. \& Kumar, V. (2013). Testes play a role in termination but not in initiation of the spring migration in the night-migratory blackheaded bunting. Animal Biology, 63, 321-329.

13. Gupta, N. J., Nanda, R. K., Das, S., Das, M. K. \& Arya, R. (2020). Night migratory songbirds exhibit metabolic ability to support high aerobic capacity during migration. ACS Omega, 5(43), 28088-28095. doi.org/10.1021/ acsomega.0c03691

14. Hen, G., Yosefi, S., Ronin, A., Einat, P., Rosenblum, C. I., Denver, R. J. \& Friedman-Einat, M. (2008). Monitoring leptin activity using the chicken leptin receptor. The Journal of Endocrinology, 197(2), 325-333. doi.org/10.1677/ JOE-08-0065

15. Henderson, L. J., Cockcroft, R. C., Kaiya, H., Boswell, T. \& Smulders, T. V. (2018). Peripherally injected ghrelin and leptin reduce food hoarding and mass gain in the coal tit (Periparus ater). Proceedings. Biological Sciences, 285 (1879), 20180417. doi.org/10.1098/rspb.2018.0417

16. Jain, N. \& Kumar, V. (1995). Changes in food intake, body weight, gonads and plasma concentrations of thyroxine, luteinizing hormone and testosterone in captive buntings exposed to natural day lengths at $29 \circ \mathrm{N}$. Journal of Biosciences, 20, 417-426.

17. Kaiya, H., Furuse, M., Miyazato, M. \& Kangawa, K. (2009). Current knowledge of the roles of ghrelin in regulating food intake and energy balance in birds. General and Comparative Endocrinology, 163(1-2), 33-38. doi.org/10.1016/j.ygcen.2008.11.008

18. Klaassen M. (1996). Metabolic constraints on longdistance migration in birds. The Journal of Experimental Biology, 199 (1), 57-64.

19. Kochan, Z., Karbowska, J. \& Meissner, W. (2006). Leptin is synthesized in the liver and adipose tissue of the dunlin (Calidris alpina). General and Comparative Endocrinology, 148(3), 336-339. doi.org/10.1016/j.ygcen.2006.04.004

20. Krause, J. S., Pérez, J. H., Meddle, S. L. \& Wingfield, J. C. (2017). Effects of short-term fasting on stress physiology, body condition, and locomotor activity in wintering male white-crowned sparrows. Physiology \& Behavior, 177, 282-290. doi.org/10.1016/j.physbeh.2017.04.026

21. Kumar, V., Singh, S., Misra, M. \& Malik, S. (2001). Effects of duration and time of food availability on photoperiodic responses in the migratory male blackheaded bunting (Emberiza melanocephala). The Journal of Experimental Biology, 204(16), 2843-2848.

22. Kurokawa, T. \& Ohkohchi, N. (2017). Platelets in liver disease, cancer and regeneration. World Journal of Gastroenterology, 23(18), 3228-3239. doi.org/10.3748/ wjg.v23.i18.3228

23. Landys, M. M., Piersma, T., Guglielmo, C. G., Jukema, J., Ramenofsky, M. \& Wingfield, J. C. (2005). Metabolic profile of long-distance migratory flight and stopover in a shorebird. Proceedings. Biological Sciences, 272(1560), 295-302. doi.org/10.1098/rspb.2004.2952

24. Lõhmus, M. \& Björklund, M. (2009). Leptin affects life history decisions in a passerine bird: a field experiment. PLOS One, 4(2), e4602. doi.org/10.1371/journal.po ne.0004602

25. Lõhmus, M., Sandberg, R., Holberton, R. L. \& Moore, F. R. (2003). Corticosterone levels in relation to migratory readiness in red-eyed vireos (Vireo olivaceus). Behavioral Ecology and Sociobiology, 54, 233-239. doi.org/10.1007/ s00265-003-0618-z

26. Lynn, S. E., Breuner, C. W. \& Wingfield, J. C. (2003). Short-term fasting affects locomotor activity, corticosterone, and corticosterone binding globulin in a migratory songbird. Hormones and Behavior, 43(1), 150-157. doi.org/10.1016/s0018-506x(02)00023-5

27. Malik, S., Rani, S. \& Kumar, V. (2004). Wavelength dependency of light-induced effects on photoperiodic clock in the migratory blackheaded bunting (Emberiza melanocephala). Chronobiology International, 21(3), 367-384. doi.org/10.1081/cbi-120038742

28. McWilliams, S. R., Guglielmo, C., Pierce, B. \& Klaassen, M. (2004). Flying, fasting, and feeding in birds during migration: a nutritional and physiological ecology perspective. Journal of Avian Biology, 35(5), 377-393. doi.org/10.1111/j.0908-8857.2004.03378.x

29. Misra, I. (2016). Mechanism of adaptation for breeding in opportunistic, atypical photosensitive and photoperiodic songbirds. PhD Thesis submitted to Delhi University, India.

30. Morton, M. L. (1967). Diurnal Feeding Patterns in White- 
crowned Sparrows, Zonotrichia leucophrys gambelii. Condor, 69(5), 491-512.

31. Myers, S., Bowden, R., Tumian, A., Bontrop, R. E., Freeman, C., MacFie, T. S., McVean, G. \& Donnelly, P. (2010). Drive against hotspot motifs in primates implicates the PRDM9 gene in meiotic recombination. Science, 327 (5967), 876-879. doi.org/10.1126/science.1182363

32. Perfito, N., Kwong, J. M., Bentley, G. E. \& Hau, M. (2008). Cue hierarchies and testicular development: is food a more potent stimulus than day length in an opportunistic breeder (Taeniopygia g. guttata)?. Hormones and Behavior, 53(4), 567-572. doi.org/10.1016/j.yhbeh.2008.01.002

33. Prabhat, A., Batra, T. \& Kumar, V. (2020). Effects of timed food availability on reproduction and metabolism in zebra finches: Molecular insights into homeostatic adaptation to food-restriction in diurnal vertebrates. Hormones and Behavior, 125, 104820. doi.org/10.1016/j.yhbeh.20 20.10 4820

34. Rees, E. C. (1982). The effect of photoperiod on the tim- ing of spring migration in the Bewick's Swan. Wildfowl, 33, 119-132.

35. Seroussi, E., Knytl, M., Pitel, F., Elleder, D., Krylov, V., Leroux, S., Morisson, M., Yosefi, S., Miyara, S., Ganesan, S., Ruzal, M., Andersson, L. \& Friedman-Einat, M. (2019). Avian Expression Patterns and Genomic Mapping Implicate Leptin in Digestion and TNF in Immunity, Suggesting That Their Interacting Adipokine Role Has Been Acquired Only in Mammals. International Journal of Molecular Sciences, 20(18), 4489. doi.org/10.3390/ijms20184489

36. William, W. N., Jr, Ceddia, R. B. \& Curi, R. (2002). Leptin controls the fate of fatty acids in isolated rat white adipocytes. The Journal of Endocrinology, 175(3), 735-744. doi.org/10.1677/joe.0.1750735

37. Yosefi, S., Hen, G., Rosenblum, C. I., Cerasale, D. J., Beaulieu, M., Criscuolo, F. \& Friedman-Einat, M. (2010). Lack of leptin activity in blood samples of Adélie penguin and bar-tailed godwit. The Journal of Endocrinology, 207 (1), 113-122. doi.org/10.1677/JOE-10-0177 